\title{
Infrared Measurements of the RF Output of 170-GHz/2-MW Coaxial Cavity Gyrotron and Its Phase Retrieval Analysis
}

\author{
Sudheer Jawla, Student Member, IEEE, Jean-Philippe Hogge, Stefano Alberti, \\ Timothy Goodman, Bernard Piosczyk, and Tomasz Rzesnicki
}

\begin{abstract}
We report the experimental results of the infrared measurements of output RF beam of the European 2-MW 170-GHz coaxial cavity gyrotron for ITER. The output beam profile is measured by the infrared thermographic technique using an infrared camera and a target material which is being irradiated by the RF output. The beam intensity was measured at several locations along the propagation direction. The data were processed for noise reduction and perspective correction and then used with the phase retrieval algorithm to yield an accurate estimate of the phase profile. The moments of the measured intensity arrays are calculated to improve the reliability of the measured infrared image data and data processing. The beam profile is also measured at specific locations in order to compare the phase calculated with the Huygens-Fresnel propagation method for fixed distance and Rayleigh-Sommerfeld diffraction integral method used for field propagation in the phase retrieval algorithm.
\end{abstract}

Index Terms-Gaussian beam, gyrotron, image denoising, infrared thermography, phase retrieval.

\section{INTRODUCTION}

$\mathbf{T}$ HE European 170-GHz/2-MW/CW coaxial cavity gyrotron for ITER [1], which is presently being tested in Lausanne, is a collaborative effort between the European research associations and a European industrial partner (Thales ED, Velizy, France). As a part of the experimental campaign during the first prototype operation of the gyrotron [2], [3], IR measurements were performed at short pulselength for the characterization of the output microwave beam profile. For this purpose, an RF box was designed as a part of the whole test facility [1] and used for the IR measurements. Since direct phase measurements are not available at such high power, one has to rely on the intensity measurements to calculate the phase associated with the RF beam which is used for the full beam analysis to couple the gyrotron RF output to the transmission

Manuscript received October 10, 2008; revised November 18, 2008. First published February 10, 2009; current version published March 11, 2009. This work was supported by the European Communities under the contract of Association between EURATOM/Switzerland, carried out within the framework of the European Fusion Development Agreement (Task agreement: TW6THHE-TFO1).

S. Jawla, J.-P. Hogge, S. Alberti, and T. Goodman are with the Centre de Recherche en Physique des Plasmas, Association EURATOM-Confédération Suisse, Ecole Polytechnique Fédérale de Lausanne, 1015 Lausanne, Switzerland (e-mail: sudheerjawla@gmail.com).

B. Piosczyk and T. Rzesnicki are with the Institut für Höchleistungsimpulsund Mikrowellentechnik, Association EURATOM-Forschungszentrum Karlsruhe (FZK), FZK, 76021 Karlsruhe, Germany.

Color versions of one or more of the figures in this paper are available online at http://ieeexplore.iee.org.

Digital Object Identifier 10.1109/TPS.2008.2011488

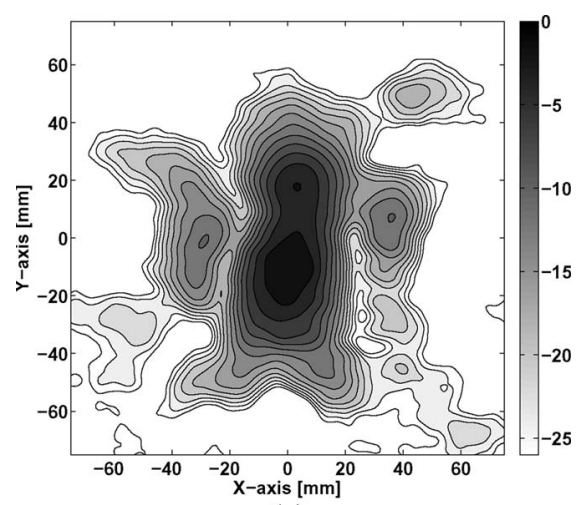

(a)

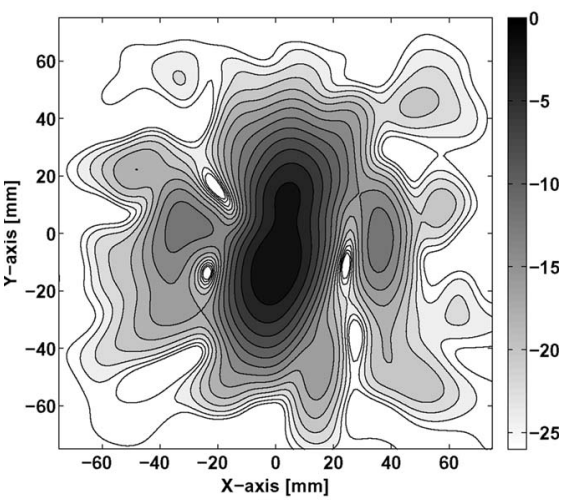

(b)

Fig. 1. Measured and simulated power distribution on the logarithmic scale at $464 \mathrm{~mm}$ from the gyrotron window plane. (a) High-power measurement. (b) Simulation with diffraction integral.

lines for an efficient transfer to the ITER electron cyclotron launcher. These high-power intensity measurements

are performed using an IR thermographic technique where a target material is irradiated with the RF output of the gyrotron, and the corresponding temperature elevation is recorded using an IR camera at some angle with respect to the microwave beam path. The phase profiles were then retrieved from the intensity profiles using the iteration methods explained in [4]-[9]. In this paper, we emphasize the measurement accuracies during the IR measurements and post-data processing, which could affect the accuracy of the reconstructed phase calculated by the iterative phase retrieval approach (IPRA). The retrieved intensity profiles calculated by the IPRA are compared with the measured and the simulated profiles [10]. In Fig. 1, a high-power IR measurement is shown in comparison with the simulation with the Forschungszentrum Karlsruhe (FZK) code 


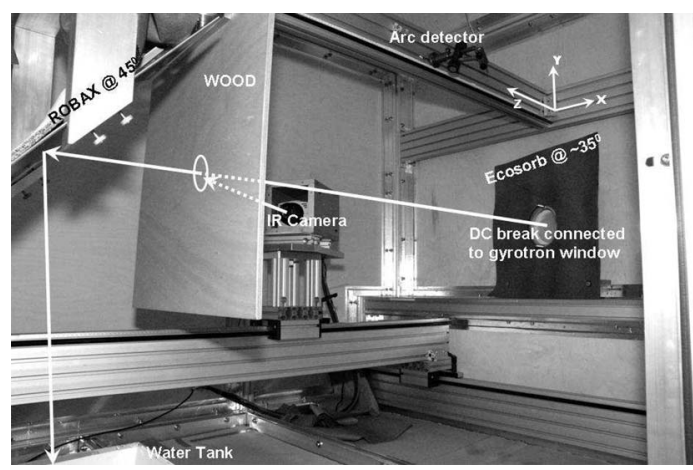

Fig. 2. Experimental setup for the high-power RF output measurements of the gyrotron. In the configuration shown, the measurement is done from the front side.

based on Kirchhoff diffraction integral. As can be seen from the power plots in the linear scale, the simulation is able to predict the behavior of the output beam. Therefore, we can make a direct comparison of the measured intensity profiles and retrieved intensity profiles with the simulated profiles. This paper, with the experimental data, provides a verification of the analysis given in [8].

This paper is organized as follows. Section II describes the experimental setup and measurement procedure for IR measurements. Section III discusses the data processing for phase reconstruction. Section IV provides a thorough discussion on the phase reconstruction using the measured intensity profiles, followed by conclusions in Section V.

\section{EXPERIMENTS}

\section{A. Experimental Setup}

A microwave leak-tight RF box, of dimension $206.5 \mathrm{~cm}$ (length) $\times 180 \mathrm{~cm}$ (width) $\times 164 \mathrm{~cm}$ (height), was designed and used to perform the free-space microwave beam-profile measurements of the gyrotron RF output. Inside the box, a target material placed on an optical bench was irradiated with the output microwave beam, and the temperature profile (or the corresponding beam-intensity pattern) was recorded using an IR camera (focal plane array of $320 \times 240$ pixels) at an angle with respect to the beam propagation direction. The setup was moved jointly along the beam direction in order to record the beam pattern at several locations from the window. As shown in Fig. 2, the DC-break (connected to the output window of the gyrotron) and its surroundings are covered with the absorbing material Eccosorb at an angle of $\sim 35^{\circ}$ in order to avoid any possibility of reflection to the target material. The IR camera is put in a magnetic shield box to avoid any perturbation of the camera electronics due to the high magnetic field of the gyrotron. A tank filled with water and a reflecting material at $45^{\circ}$ was used behind the target as a beam dump. The inside of the box was made with wood and covered with an absorbing cloth to minimize any possible reflections.

\section{B. Infrared Measurement Procedure}

During the beam-profile measurements, the gyrotron operates below its nominal parameters; the beam current was set

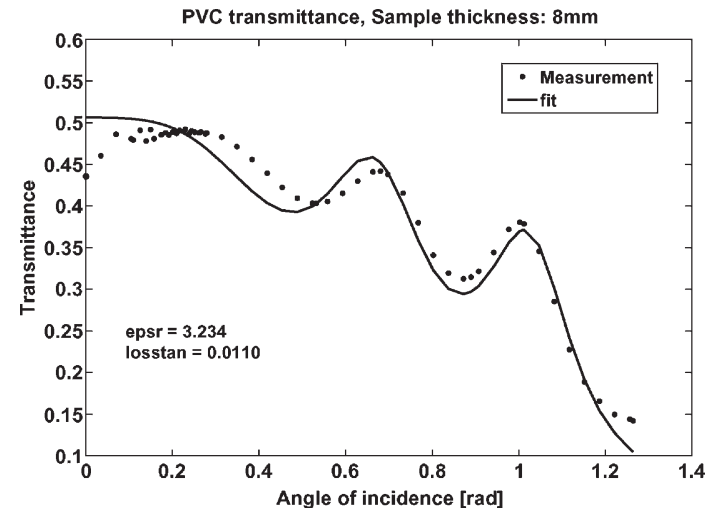

Fig. 3. PVC transmittance measured at $170 \mathrm{GHz}$ with the free-space permittivity measurement method. Sample thickness is $8 \mathrm{~mm}$.

to $66 \mathrm{~A}$ and the cathode voltage to $78 \mathrm{kV}$ for a pulse duration of about $1 \mathrm{~ms}$. A frequency diagnostic was also used to verify the correct excitation of the nominal gyrotron operating mode $\mathrm{TE}_{34,19}$. During the measurements, we explored a number of ways to get an accurate beam-profile measurement at every location from the gyrotron window. This includes the correct choice of the target material (to avoid interference effects due to reflections), proper alignment of the target, correct positions of the measurement locations [9], etc. To optimize the correct target material, we tested four different materials, namely, Robax, PVC, Kapton, and wood. The complex permittivity of PVC was measured at the frequency of $170 \mathrm{GHz}$ using a freespace permittivity method [11] with samples of three different thicknesses 4,6 , and $8 \mathrm{~mm}$, respectively. The material was then optimized for the thickness to be used at $170 \mathrm{GHz}$ in order to minimize the back reflection to the gyrotron window and to maximize the absorption. Fig. 3 shows the measured transmittance as a function of the incidence angle for a sample thickness of $8 \mathrm{~mm}$ for PVC. The permittivity and loss tangent calculated by the measured transmittance are $\varepsilon_{r}=3.234$ and $\tan \delta=1.1 \times 10^{-2}$, respectively. The permittivity of Robax at $170 \mathrm{GHz}$ was found from the literature, and then, an optimized thickness was calculated for minimum reflection at this frequency. A wrong selection of the thickness can give rise to a large back reflection inside the gyrotron, which can seriously affect the whole operation of the gyrotron. The optimized thickness for PVC was $5.4 \mathrm{~mm}$. For Robax, the optimized thickness was $4.1 \mathrm{~mm}$; however, as it is difficult to machine, we used $4 \mathrm{~mm}$ (commercially available). For wood, we used $12 \mathrm{~mm}$ and for Kapton, $0.1 \mathrm{~mm}$ (commercially available foil). Robax and PVC were also checked for temperature linearity with respect to IR camera response for the operating power range (output beam power). It was found that PVC (5.4 mm thick) behaves linearly up to $65{ }^{\circ} \mathrm{C}$, while Robax showed a higher linear temperature sustainability up to $125^{\circ} \mathrm{C}$. The operating temperature range during the measurements was $25^{\circ} \mathrm{C}-40{ }^{\circ} \mathrm{C}$ for PVC and $25{ }^{\circ} \mathrm{C}-60{ }^{\circ} \mathrm{C}$ for Robax. The temperature was measured using an IR thermometer. The measurements were then performed with all four targets. The beam-intensity profile was also measured from both directions-front and back sides on the target. The alignment of the target material was checked, with respect to the window, using a laser. The measurements should be done within the Rayleigh range and as explained 


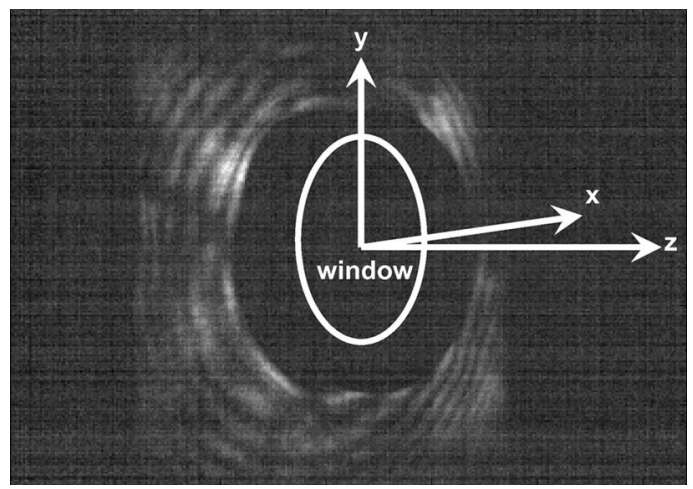

Fig. 4. Interference pattern at the window. The image was recorded in the $x-z$ plane at an angle with respect to the beam propagation direction $z$.

in [8] and [9]; the reconstruction algorithm will result in an accurate phase profile if the measurements are performed as close as possible to the beam-waist location (also explained in Section IV-B). We therefore tried to measure the beam profile as close as possible (at $441 \mathrm{~mm}$ ) to the gyrotron window. In both configurations (front- and back-side measurements), the IR camera was placed at an angle of $35^{\circ}$ with respect to the beam propagation direction. The recorded images were then corrected for perspective.

For the measurements close to the window, strong interference effects were observed. These interference effects are due to the reflections between the target material/window and its support and the Fabry-Parot effects inside the target material itself. Fig. 4 shows an interference pattern at the window and its support. The image is recorded in the $x-z$ plane at an angle with respect to the propagation axis $z$. To minimize the reflections due to window support, we placed an absorbing material covering the window surroundings at an angle of $\sim 35^{\circ}$ close to the window, as shown in Fig. 2.

\section{INFRARED IMAGE DATA PROCESSING FOR PHASE RECONSTRUCTION}

\section{A. IR Image Data Denoising}

To model phase retrieval realistically, one must consider the effect of noise inherent in making the IR measurements of microwave beam intensity. Due to this inherent noise, raw data, in themselves, are not appropriate for phase reconstruction. Practically speaking, it is observed that IR images are mainly degraded by fixed-pattern noise (FPN) due to pixel reading procedure, presence of dead pixels in the focal-plane-array matrix of the camera, radial distortion due to noncollinear image points with respect to the optical center, etc. Some of these noise effects can be removed to a large extent, e.g., in our case, FPN is cancelled out by subtracting the uniform background image of the target taken before the microwave irradiation. A map of dead pixels is generally clear in the image when data are taken at relatively high temperatures. To correct the dead pixel matrix, pixels are replaced by the average values of the neighboring pixels. For a bigger cluster of inactive pixels, an object-oriented transform coding technique [12] is used.

During the measurements, the images are recorded in a transverse plane at some angle, with respect to the direction of propagation of the output microwave beam, and thereafter, perspective correction is made for each measured plane by geometrical transformation or by prior knowledge of the angles between different measuring objects. After the perspective correction, data are corrected for the FPN and the inactive pixel matrix, as mentioned earlier. Further, the image pattern enhancement, i.e., improving signal-to-noise ratio is performed by using an image denoising algorithm before the data can be used efficiently for the phase reconstruction algorithm. This is done in the following way. Since measurements are done on a target material with a larger size compared with the beam size, therefore, to a reasonable approximation, one can assume that the field at the boundary is close to zero and that the values of the field at the boundary corresponds to the level of noise in the image (after FPN correction). This can be a reliable approximation since it is difficult, in this case, to properly model the noise and its parameters.

Considering the amount of noise by its standard deviation, we applied some existing image denoising algorithms to improve the quality of images. These are the following:

1) image denoising by total variance minimization [13], [14];

2) denoising by a nonlocal means algorithm [15];

3) filtering in Fourier space using a Fermi-Dirac function [16];

4) moving average filter (boxcar filter).

The details for each one can be found in the references cited. Since image denoising is a smoothing process, it affects the position of the beam's center of gravity and size, depending on different smoothing techniques. Therefore, one should choose the appropriate filtering method depending on the image data and the amount of noise in it. In our case, we applied the method of filtering in Fourier space using a Fermi-Dirac function.

We also compared the beam-intensity profiles measured from the front and back sides of the target material, and all the intensity profiles were then aligned so that they could be efficiently used in the phase retrieval algorithm. It is also worth mentioning here that we assumed that the gyrotron behaves identically during every shot for the measurements at different positions and delivers the same amount of power at the same operating parameters. Therefore, we use the same filtering techniques and similar statistics of noise at every plane location during IR data processing. Different powers delivered by the gyrotron during different shots could lead to inconsistency in beam pattern measurement and, therefore, the filtering of the recorded IR images. With a Robax target, the IR camera was able to record the IR images of the output beam intensity down to $\sim-25 \mathrm{~dB}$ and after appropriate filtering (small gain, in decibels); the IR image data could be analyzed down to $\sim-30 \mathrm{~dB}$.

\section{B. Measurements Done From Front and Back Sides With Different Target Materials}

We checked the accuracy of the beam-intensity profiles by comparing the beam size when the measurements were done from both the front and back sides of the target. The effective beamwidth along the transverse direction $x$ is calculated as

$$
W_{x}=2\left(\left\langle x^{2}\right\rangle-\langle x\rangle^{2}\right)^{1 / 2}
$$


TABLE I

COMPARISON OF BEAM CENTROID AND EFFECTIVE BEAMWIDTH FOR ROBAX AND PVC AT TWO DIFFERENT LOCATIONS FROM THE OUtPUT Window WHEN MEASURED FROM FRONT AND BACK Sides; Units ARE IN MiLLIMETERS

\begin{tabular}{|c|c|c|c|c|}
\hline \multicolumn{5}{|c|}{ PVC } \\
\hline Distance & $<x>$ & $\langle y\rangle$ & $W_{x}$ & $W_{y}$ \\
\hline $\begin{array}{l}1122 \mathrm{~mm} \\
\text { front side } \\
\text { back side }\end{array}$ & $\begin{array}{l}-1.561 \\
-0.168\end{array}$ & $\begin{array}{l}-6.015 \\
-6.028\end{array}$ & $\begin{array}{l}77.357 \\
69.236\end{array}$ & $\begin{array}{l}73.573 \\
67.397\end{array}$ \\
\hline $\begin{array}{l}1282 \mathrm{~mm} \\
\text { front side } \\
\text { back side }\end{array}$ & $\begin{array}{l}-3.623 \\
-2.510\end{array}$ & $\begin{array}{l}-3.561 \\
-4.064\end{array}$ & $\begin{array}{l}85.579 \\
74.332\end{array}$ & $\begin{array}{l}80.776 \\
73.216\end{array}$ \\
\hline \multicolumn{5}{|c|}{ ROBAX } \\
\hline Distance & $<x>$ & $<x>$ & $W_{x}$ & $W_{y}$ \\
\hline $\begin{array}{l}1122 \mathrm{~mm} \\
\text { front side } \\
\text { back side }\end{array}$ & $\begin{array}{l}-2.357 \\
-1.915\end{array}$ & $\begin{array}{l}-5.652 \\
-4.980\end{array}$ & $\begin{array}{l}78.054 \\
74.907\end{array}$ & $\begin{array}{l}72.031 \\
73.104\end{array}$ \\
\hline $\begin{array}{l}1282 \mathrm{~mm} \\
\text { front side } \\
\text { back side }\end{array}$ & $\begin{array}{l}-2.623 \\
-2.026\end{array}$ & $\begin{array}{l}-3.871 \\
-2.556\end{array}$ & $\begin{array}{l}84.331 \\
80.603\end{array}$ & $\begin{array}{l}76.989 \\
77.510\end{array}$ \\
\hline
\end{tabular}

where $\langle x\rangle$ is the center of the power density coordinate and is given by the moment method as

$$
\left\langle x^{n}\right\rangle=\frac{\iint\left(x^{n}\right) I(x, y) d x d y}{\iint I(x, y) d x d y}
$$

and $I(x, y)$ represents the intensity of the beam. A similar expression holds for the $y$-direction. The geometry for $x$ and $y$ is shown in Figs. 2 and 4.

The comparison is made in Table I for the Robax target of 4-mm thickness and for PVC of 5.4-mm thickness for two positions. The intensity is measured from both directions for the two targets at the positions of 1122 and $1282 \mathrm{~mm}$ from the gyrotron window at the same operating conditions and geometric configurations. The beam centroid for the two materials is also compared simultaneously. The small difference in the beam centroid for PVC and Robax can be due to the slight misalignment during the change of the target material when performing the measurements. The thermal diffusion coefficient of PVC is larger than Robax along the thickness, and one should see more difference in $W_{x, y}$ in the case of Robax than of the PVC; however, it is found that PVC demonstrates the larger difference in the effective beam waist when measured from the front and back sides. The reason can be due to the fact that Robax is transparent to IR radiation and the camera receives a superposed thermal radiation from along the whole thickness of the material. Therefore, with PVC, one should do the measurements from the front side in order to be more accurate in terms of the beam size or one should use the Robax material which gives almost the same beam size from both directions.

\section{Alignment}

For experimentally measured intensity patterns, one should strictly align the field amplitude profiles with an accuracy of a wavelength in order to avoid errors in the phase retrieval method. For this purpose, the expression for field propagation must be corrected for the shift in the beam center of gravity at each plane location for experimentally measured profiles. For an arbitrary-shaped beam propagating in a uniform media, the beam center-of-gravity coordinates vary linearly with $z$ [5];

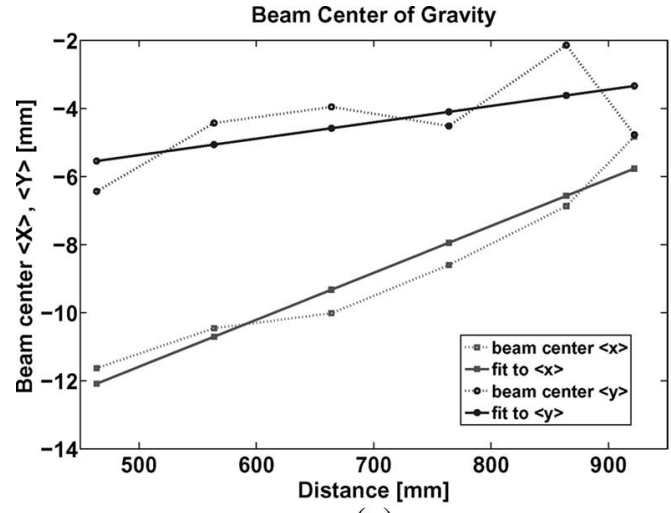

(a)

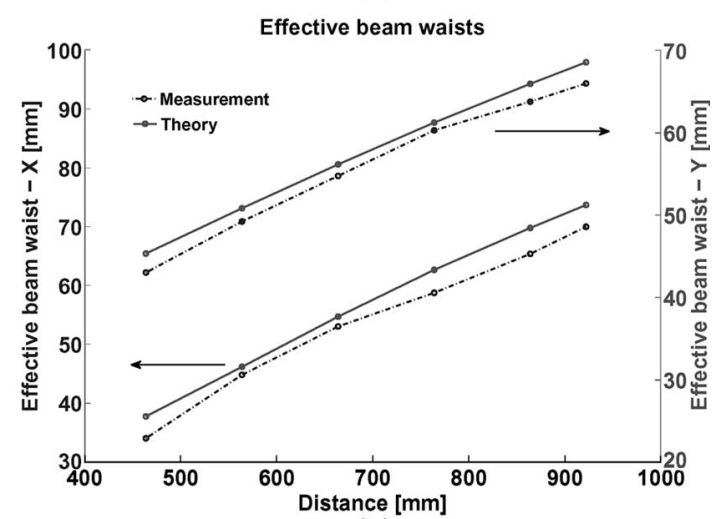

(b)

Fig. 5. (a) First-order moment evolution along the $x$ - and $y$-directions of the output beam. The tilt calculated by $\langle x\rangle$ and $\langle y\rangle$ are $0.79^{\circ}$ and $0.28^{\circ}$ in the $x$ - and $y$-directions, respectively. (b) Effective beam waist in the $x$ - and $y$-directions plotted along with the beam waists calculated by theoretical beam profiles. The coordinate geometry is shown in Figs. 2 and 4.

therefore, the centroid of beam intensity at every measured location is calculated using (2), and then, the beam profiles were aligned using the Fourier shift theorem (i.e., Fourier sinc interpolation via simple phase adjustments in the frequency domain) in order to compensate for the misalignment during the measurements. The beam patterns were aligned using the values calculated by the linear fit values of the centroid shown in Fig. 5(a). The aligned beam profiles were than used in the IPRA for the phase reconstruction. To implement the shift in the beam center of gravity in the IPRA, the correction term due to $\langle x\rangle$ and $\langle y\rangle$ can also be introduced in the plane wave propagation equation [8], [17], using the Fourier shift theorem, as

$$
\begin{aligned}
u_{j}(x, y)=\mathcal{F}^{-1}\left\{\mathcal{F}\left[u_{i}(x, y)\right]\right. & \times \exp \left[i\left(z_{j}-z_{i}\right) k_{z}\right] \\
& \times \underbrace{\exp \left[i\left(k_{x}\langle x\rangle+k_{y}\langle y\rangle\right)\right]}_{\text {correction factor }}\}
\end{aligned}
$$

where $u_{i}(x, y)$ and $u_{j}(x, y)$ are the field in the input plane at $z_{i}$ and the output plane $z_{j}$ and $\mathcal{F}$ and $\mathcal{F}^{-1}$ represent the Fourier transform and its inverse, respectively. The plots of the beam center $\langle x\rangle$ and $\langle y\rangle$ and the effective beamwidth for the measured positions of the IR images are shown in Fig. 5. The beamwidths calculated from the theoretical field profiles are also plotted with the measured ones. Fig. 5 shows that the beam is propagating at an angle with respect to the optical 

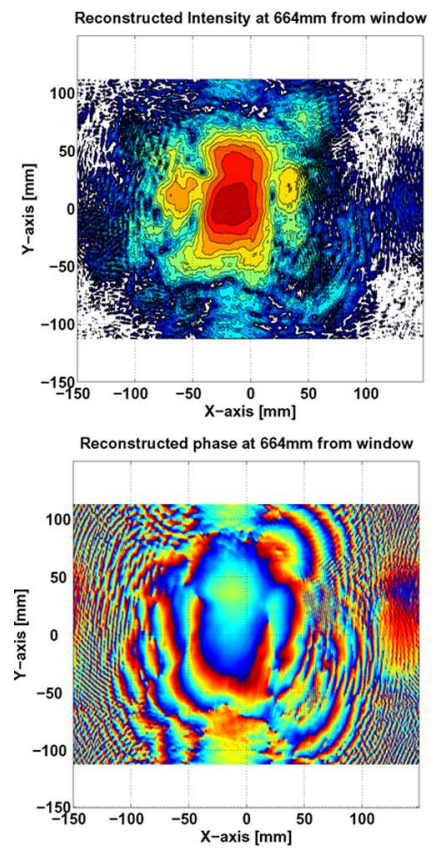

(a)

HFP: $\mathrm{L}=300 \mathrm{~mm} \times 225 \mathrm{~mm} ; \mathrm{M}=512$, $\mathrm{N}=288 ; \Delta \mathrm{x}=\Delta \mathrm{y}$ Forward propagation: $\quad 1 \rightarrow 2 \rightarrow 3$ Backward propagation: $3 \rightarrow 2 \rightarrow 1$
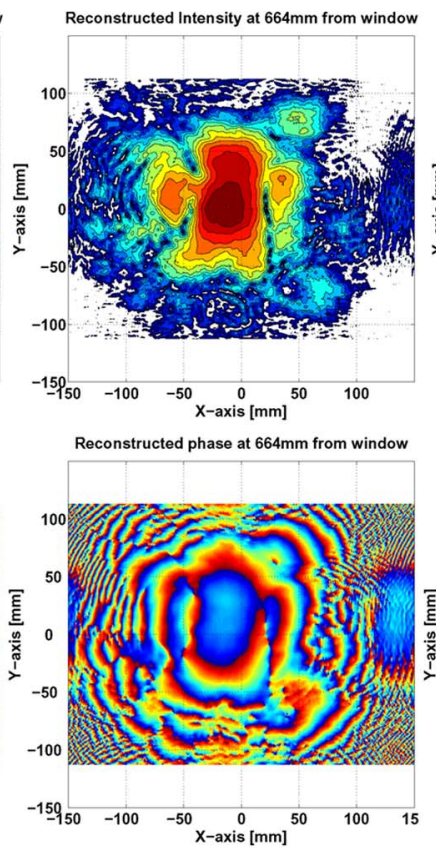

(b)

HFP: $\mathrm{L}=300 \mathrm{~mm} \times 225 \mathrm{~mm} ; \mathrm{M}=320$ $\mathrm{N}=240 ; \Delta \mathrm{x}=\Delta \mathrm{y}$.

Forward propagation: $\quad 1 \rightarrow 2 \rightarrow 3$

Backward propagation: $3 \rightarrow 1$
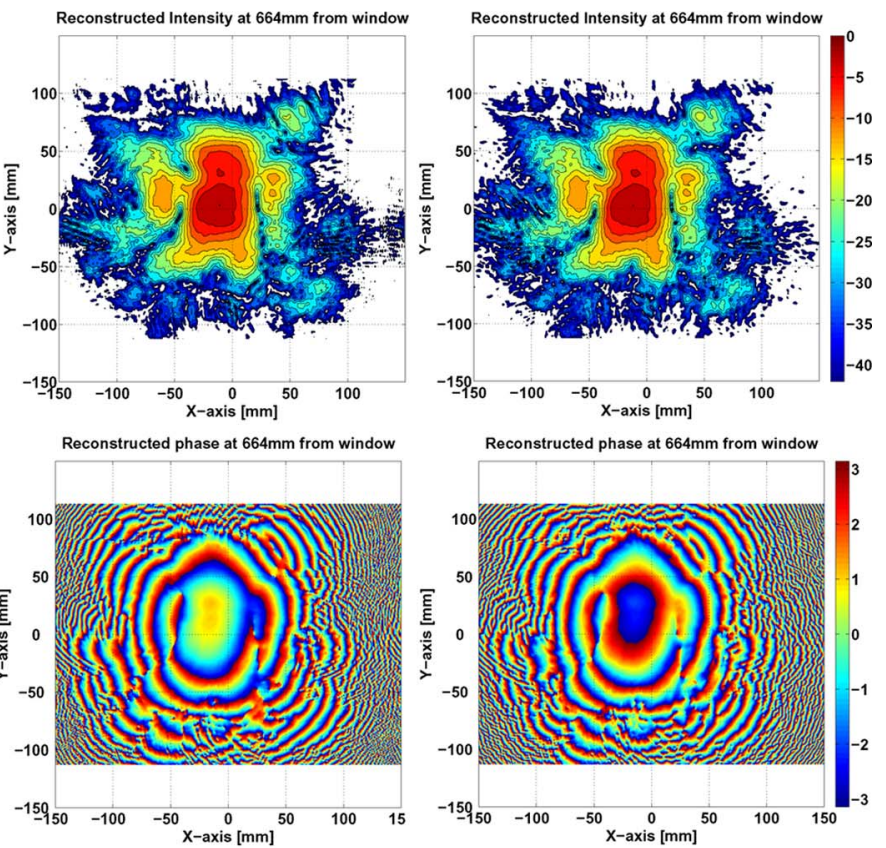

(c)

HFP: $\mathrm{L}=300 \mathrm{~mm} \times 300 \mathrm{~mm} ; \mathrm{M}=512$ $\mathrm{N}=512 ; \Delta \mathrm{x}=\Delta \mathrm{y}$. Forward propagation: $\quad 1 \rightarrow 2 \rightarrow 3$ Backward propagation: $3 \rightarrow 1$

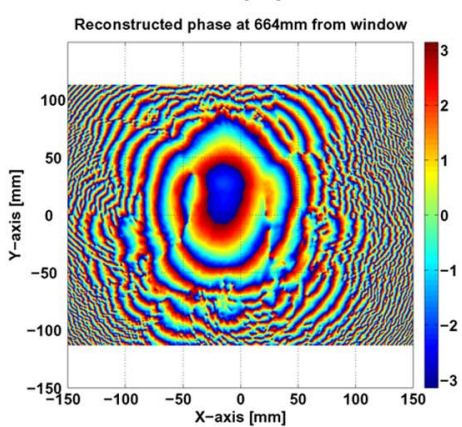

(d)

RSDI: $\mathrm{L}=300 \mathrm{~mm} \times 225 \mathrm{~mm} ; \mathrm{M}=320$ $\mathrm{N}=240 ; \Delta \mathrm{x}=\Delta \mathrm{y}$. Forward propagation: $\quad 1 \rightarrow 2 \rightarrow 3$ Backward propagation: $3 \rightarrow 1$

Fig. 6. Reconstructed beam-intensity patterns and phases at $664 \mathrm{~mm}$ from the output window. The IPRA was performed with the (a, b, and c) HFP method with three different cases of sampling points and plane sizes and with the (d) RSDI method. The isomagnitude contours of the intensity patterns are at every $-3 \mathrm{~dB}$ from the peak intensity value, and the phase is wrapped within $2 \pi$ modulo. (c) Reconstructed field calculated by the IPRA using the HFP method following the condition of propagation, i.e., $z=\left(M \Delta x^{2}\right) / \lambda$, and one can see that there are no effects of replication and modulation. Moreover, the RSDI provides an equally good result, as shown in (d).

axis (defined to be perpendicular to the window). The tilts calculated by $\langle x\rangle$ and $\langle y\rangle$ are $0.79^{\circ}$ and $0.28^{\circ}$ in the $x$ - and $y$-directions, respectively. During the measurements, the target plane was aligned with respect to the output window plane, and the beam centers are calculated by the measured profiles defining the reference position $(0,0)$ at the window axis. It can be concluded from the beam center of gravity that the beam is offset at the window position and approaching the geometrical axis of the gyrotron window.

\section{Phase Retrieval Analysis}

The aforementioned measurement procedure and the necessary preprocessing of the IR data enables us to discuss various parameters associated with the IPRA and their effects on the reconstructed intensity and phase profiles. The phase profiles were retrieved from the measured intensity profiles using the IPRA, which has been discussed in detail in [8] in terms of field propagation schemes and sampling effects and other parameters which affect the convergence of the algorithm and influence the retrieved phase.

\section{A. Effect of Different Propagation Schemes, Number of Sampling Points, and Grid Step Size}

Here, we compare two methods of field propagation when used in the IPRA, namely, the Rayleigh-Sommerfeld diffraction integral (RSDI) and the Huygens-Fresnel propagation (HFP), both calculated using FFT and a convolution method.
For an accurate HFP method, measurements were made at specific locations, which satisfy the condition of propagation explained in [8] and [9], as

$$
z_{i \rightarrow j}=\frac{M(\Delta x)^{2}}{\lambda}
$$

The aliasing effects included by the discretization can be avoided in the case of HFP if one follows this condition. Here, $\Delta x$ is the grid step size on a square plane; $M$ is the number of sampling points, and $\lambda$ is the wavelength. Since the IR camera records the image on a rectangular aperture size $320 \times 240$, one needs to correct the condition of propagation for a rectangular plane size; therefore, in order to make $z_{i \rightarrow j ; x}=z_{i \rightarrow j ; y}$, one should have

$$
N=M \frac{L_{y}^{2}}{L_{x}^{2}}
$$

where $L_{x}$ and $L_{y}$ are the dimensions of the measurement plane and $M$ and $N$ represent the number of sampling points in the $x$ - and $y$-directions, respectively. The recorded data after preprocessing were interpolated to different grid sizes, based on (5). Fig. 6 shows the reconstructed field intensity and phase profiles calculated using RSDI and HFP as the propagation method in the IPRA. The isomagnitude contours of the intensities are plotted at every $-3-\mathrm{dB}$ increment from the peak intensity, and the phase is wrapped in a $2 \pi$ modulo and shown in radians. The reconstruction is analyzed using different plane and grid step sizes. The IPRA is performed using three 
planes at $z=464 / 564 / 664 \mathrm{~mm}$ from the output window of the gyrotron, keeping a constant separation of $100 \mathrm{~mm}$ between the three planes. The reconstructed intensity profiles are compared with the measured intensity profiles at the third plane at $z=$ $664 \mathrm{~mm}$. Fig. 6(a)-(c) shows the reconstructed intensity and phase profiles at $664 \mathrm{~mm}$ for three different cases of the plane and grid step sizes [based on (5)] in the case of HFP. Fig. 6(d) shows a comparison of the two propagation schemes, namely, the RSDI and the HFP.

1) In Fig. 6(a), the plane size is $300 \mathrm{~mm} \times 225 \mathrm{~mm}$, and the field is interpolated to $512 \times 288$ sampling points, using a mesh spacing of $\Delta x \neq \Delta y$ and $M>N$ in order to satisfy (5). The field is propagated to the next consecutive planes in the forward direction as $1 \rightarrow 2 \rightarrow 3$ and in the backward direction as $3 \rightarrow 2 \rightarrow 1$.

2) Fig. 6(b) shows the reconstructed intensity/phase profile in case of violating the condition of propagation in both directions. In this case, the same number of sampling points $320 \times 240$ (as recorded by the IR camera) are used on a plane size, similar to the previous case thus using a square grid step size $\Delta x=\Delta y$ and $M>N$ on a rectangular plane. Therefore, in this case, we are following the condition of propagation in both directions, and hence, the effects of replication and modulation are shown in the intensity and phase profiles in Fig. 6(b). The field was propagated in the following way: forward $1 \rightarrow 2 \rightarrow 3$ and backward $3 \rightarrow 1$.

3) In Fig. 6(c), the field is interpolated to a square grid for $M=N=512$ and a plane size of $300 \mathrm{~mm} \times 300 \mathrm{~mm}$, and thus, using a square grid step size in both $x$ - and $y$-directions, $\Delta x=\Delta y$. The field is propagated as follows: forward $1 \rightarrow 2 \rightarrow 3$ and backward $3 \rightarrow 1$. During the backward propagation of the field to the first plane, it is interpolated to a new grid at every iteration in order to follow the condition of propagation. This is explained in [8].

4) In Fig. 6(d), the intensity and phase profiles were retrieved using RSDI as the propagation method in the IPRA. We used the same number of sampling points, as recorded by the camera, and the same plane size of $300 \mathrm{~mm} \times 225 \mathrm{~mm}$; therefore, no interpolation to a new grid is done. The field is propagated for $1 \rightarrow 2 \rightarrow 3$ in the forward direction and $3 \rightarrow 1$ in the backward direction.

As can be clearly seen from the comparison, the RSDI as a propagation scheme in the IPRA also yields more accurate results than the previous cases shown in Fig. 6(a) and (b). From the results shown in Fig. 6(d), one can verify that we are in the regions where the effects of the higher order aberration terms in the discretization analysis of RSDI [8] are not significant. The HFP method also produces an equally good result if the condition of propagation is followed properly (5) and the grid step size is square. The reconstructed intensity profiles in Fig. 6(c) and (d) show a very good visual agreement with the measured intensity profile shown in Fig. 7. Fig. 7 also shows the theoretical intensity and phase profiles, along with the measured intensity profile at $664 \mathrm{~mm}$, from the output window in order to compare with the reconstructed field profiles

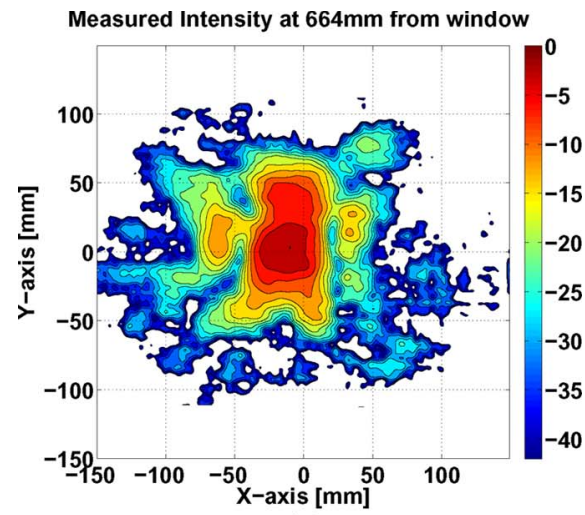

(a)

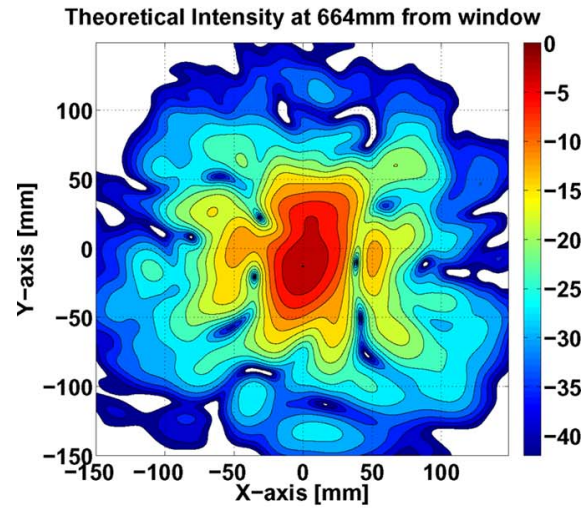

(b)

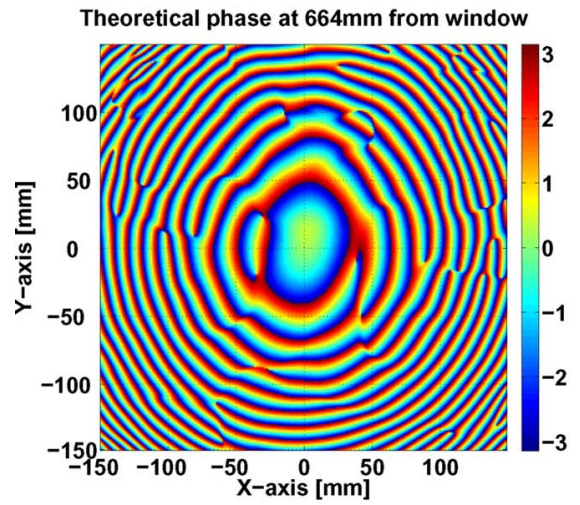

(c)

Fig. 7. (a) Measured beam-intensity profile at $664 \mathrm{~mm}$. The isomagnitude contours are at every $-3 \mathrm{~dB}$ from the peak intensity. (b) and (c) Theoretical beam-intensity and phase profiles at $664 \mathrm{~mm}$ from the gyrotron window. Figures are shown to compare the measured field intensity and reconstructed field intensity and phase (shown in Fig. 6) with theoretical field intensity and phase.

of Fig. 6. The comparison is done on the scale down to $-42 \mathrm{~dB}$ because the theoretical intensity has a dynamics up to $\sim-42 \mathrm{~dB}$ and the measured intensity (after filtering) has a dynamics level of $-30 \mathrm{~dB}$ approximately. A comparison between the measured and the theoretical field patterns can be explained in the form of a correlation function defined as

$$
C C F=\sum_{i, j} u_{r}(i, j) u_{m}(i, j)
$$

Here, $u_{r}(i, j)$ is assigned the modulus of the normalized reconstructed field amplitude, and $u_{m}(i, j)$ is the modulus of the normalized measured field amplitude. The cross correlation 


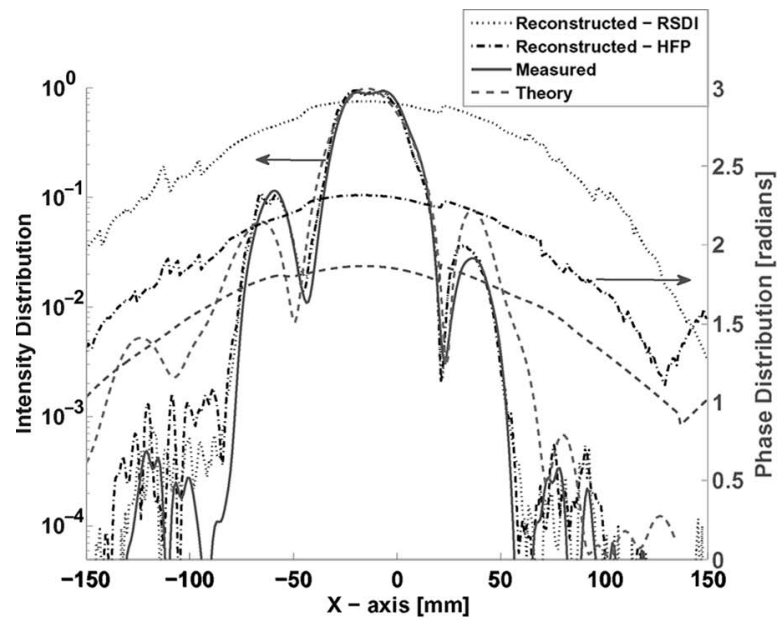

(a)

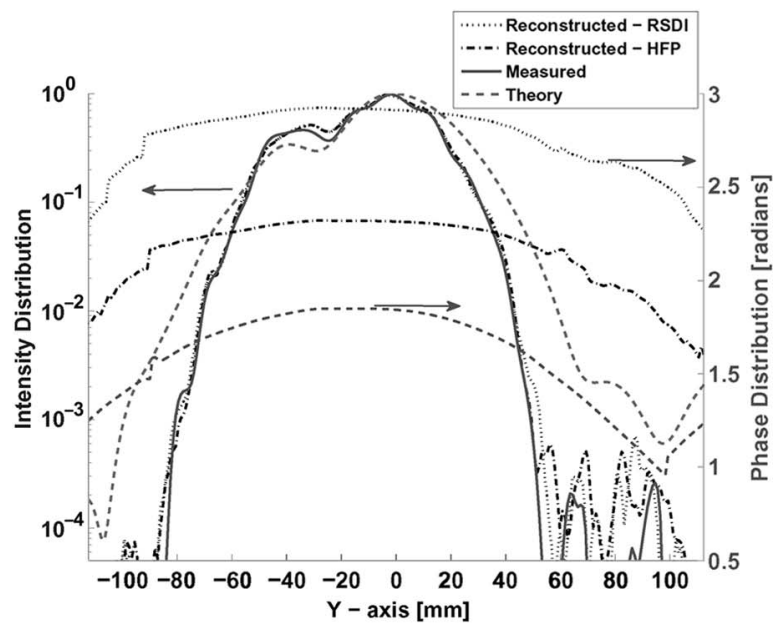

(b)

Fig. 8. (a) and (b) One-dimensional intensity (on logarithmic scale) and phase distribution (in radians) in the $x$ - and $y$-directions along the beam centroid $\langle x\rangle$ and $\langle y\rangle$, respectively, at $664 \mathrm{~mm}$ from the output window. The figure plots the theoretical, measured, and reconstructed (using HFP for fixed distance and RSDI) intensity profiles, along with the theoretical and reconstructed phase profiles. The reconstructed 2-D profiles for these two cases are shown in Fig. 6(c) and (d). The phase curvatures are consistent with the theoretical phase profile.

function $(C C F)$ is normalized such that $0 \leq C C F \leq 1$. An excellent correlation is found between the measured and theoretical profiles, apart from visual comparison shown in Fig. 7. The calculated $C C F$ for the two intensities is $90.62 \%$. A more detailed comparison between the last two cases of Fig. 6(c) and (d) is shown in Fig. 8, where the 2-D plots of reconstructed intensity and unwrapped phase are plotted, along with the measured intensity and theoretical intensity/phase. The intensity and phase profiles are shown at the beam center-ofgravity lines corresponding to $\langle x\rangle=$ const. and $\langle y\rangle=$ const. The intensity profiles are shown on a logarithmic scale down to $\sim-40 \mathrm{~dB}$, and the phase is shown in radians. Both methods of propagation, the RSDI and HFP (following the condition of propagation and a square sampling interval), produce the reconstructed intensity profiles in excellent agreement with the measured intensity down to a level of $\sim-33 \mathrm{~dB}$ below the central peak. One of the reasons for the small departure of the measured intensity from the theoretical intensity in terms of the broadening around the central peak can be understood due to the filtering. Moreover, the phase curvatures calculated by the two methods are in a good agreement with each other and with the theoretical phase profile (to mention, a constant value can always be added to the phase value to match the three phase profiles shown in Fig. 8). The phase profiles retrieved from the two methods are consistent. It is worth mentioning here that an accurate phase can be retrieved from the measured intensity profiles with either of the two methods of RSDI/HFP if the field intensity is almost zero $(\sim 60 \mathrm{~dB})$ at the boundary of the plane. This could be achieved if the measurements of the intensity profiles are performed on a larger plane size with respect to the effective beam dimension and the IR camera is placed at a position such that it could capture the image of the entire measurement plane. This is also important from the point of view of the filtering of the recorded IR images because the filtering does not differentiate between the noise and small details of the image and removes them. Therefore, the field in the outskirts of the main lobe, i.e., down to $\sim 30 \mathrm{~dB}$ or more, is removed in the filtering, as shown in Figs. 1(a) and (b) and 7(a) and (b), where the measured intensity profile is in excellent agreement with the theoretical intensity profile up to $\sim-27 \mathrm{~dB}$.

The goodness of the reconstructed results is analyzed by studying the convergence of the reconstruction process during the IPRA. The convergence criteria of the aforementioned reconstruction cases are shown in Figs. 9 and 10. Fig. 9 shows the reconstruction efficiency calculated in the form of the CCF defined by (6). For the RSDI and HFP with a square grid [Fig. 6(c)], the reconstruction efficiency reaches a value of $\sim 99.4 \%$ for plane 1 at $464 \mathrm{~mm}$ and $99.1 \%$ for the plane at $664 \mathrm{~mm}$ and saturates to almost a constant value, while in the other two cases, the $C C F$ is fluctuating and does not saturate. This can be verified by calculating the derivative of the error function during each iteration, which is shown in Fig. 10. The error function is defined by summing the squared difference of the calculated modulus of the theoretical $E_{\text {theory }}(i, j)$ and reconstructed $E_{\mathrm{rec}}(i, j)$ fields at every point of the transverse plane as

$$
\text { error }=\frac{\sum_{i, j}\left(\left|E_{\text {theory }}(i, j)\right|-\left|E_{\text {rec }}(i, j)\right|\right)^{2}}{\sum_{i, j}\left(E_{\text {theory }}(i, j)\right)^{2}} .
$$

The iteration process is stopped after 5000 steps for the case of Fig. 6(a) and after 2000 steps for the cases of Fig. 6(b)-(d). The computational time is also worth mentioning; it took approximately 60 min in MATLAB on a desktop PC (running on Windows with $2.0 \mathrm{~GB}$ of RAM and a $2.00-\mathrm{GHz}$ processor) for the IPRA using the HFP method for Fig. 9(c) (2000 iterations) and approximately $124 \mathrm{~min}$ for Fig. 9(d) (2000 iterations), where we used the RSDI method for field propagation in the IPRA. This difference is due to the fact that the RSDI was calculated using a zero padding technique; therefore, the number of sampling points was large when compared with HFP. The $C C F$ shows an almost periodic behavior for the first two cases while, on the other hand, it converges to an almost constant value for the last two cases after a sharp increase in the reconstruction efficiency of around 775 for the HFP and 


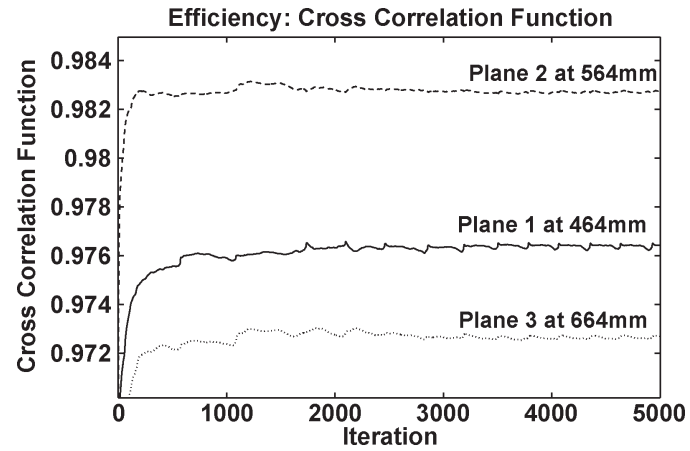

(a)

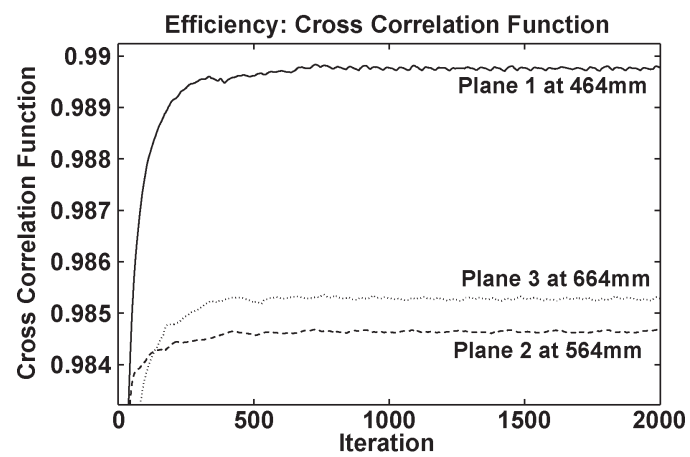

(b)

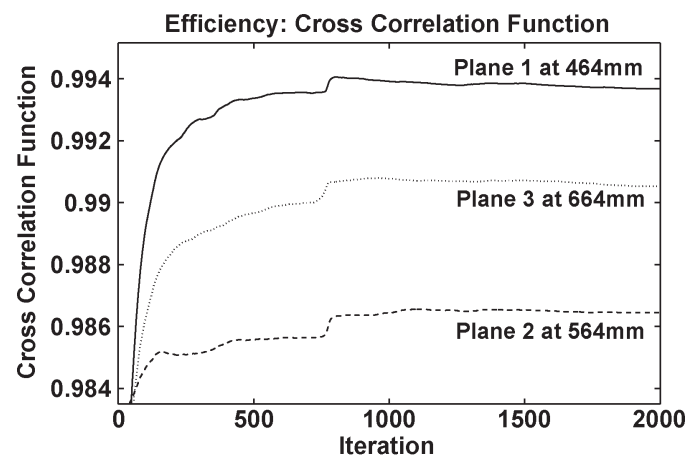

(c)

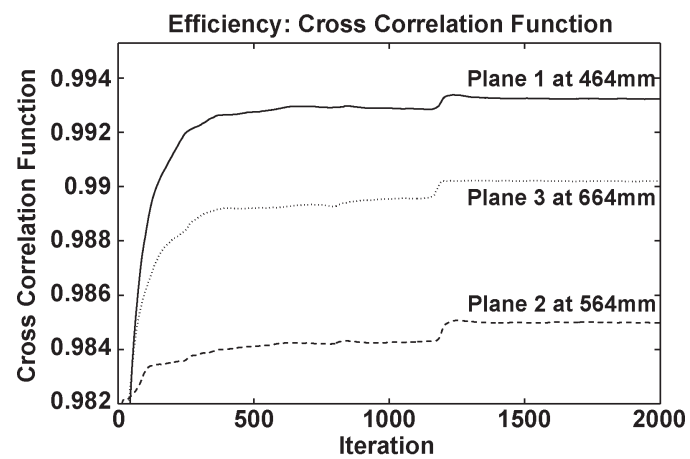

(d)

Fig. 9. Efficiency of reconstruction calculated by CCF during the IPRA. (a), (b), and (c) correspond to the different cases of HFP, while (d) is for the RSDI method.

around 1200 for the RSDI case. The convergence is faster and more accurate in the case of HFP; moreover, the efficiency of reconstruction reaches a higher value than that of the RSDI.

The reconstructed fields were also checked by propagating them to $764 \mathrm{~mm}$ (from the gyrotron output window), where the propagated intensity and phase were compared with the

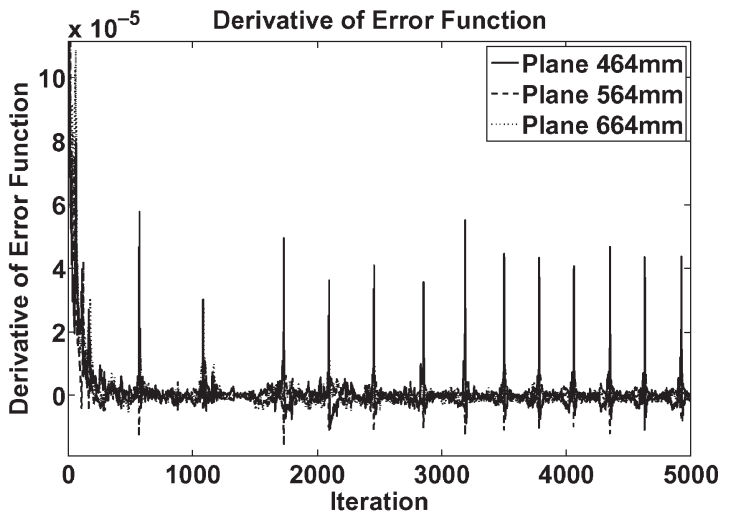

(a)

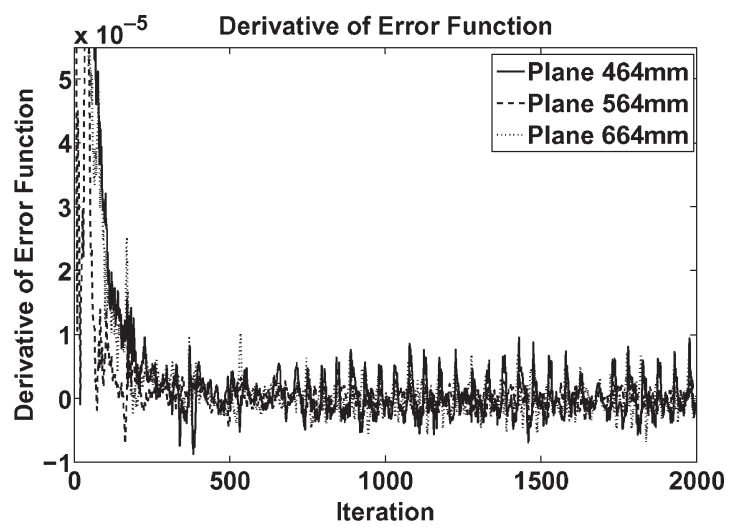

(b)

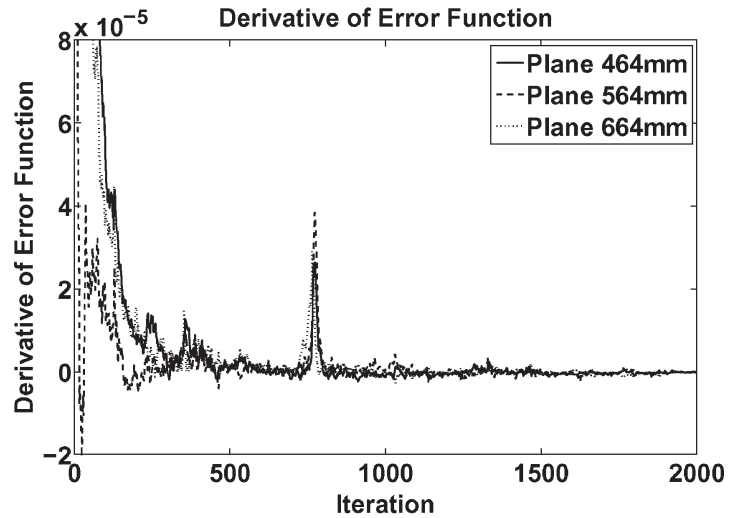

(c)

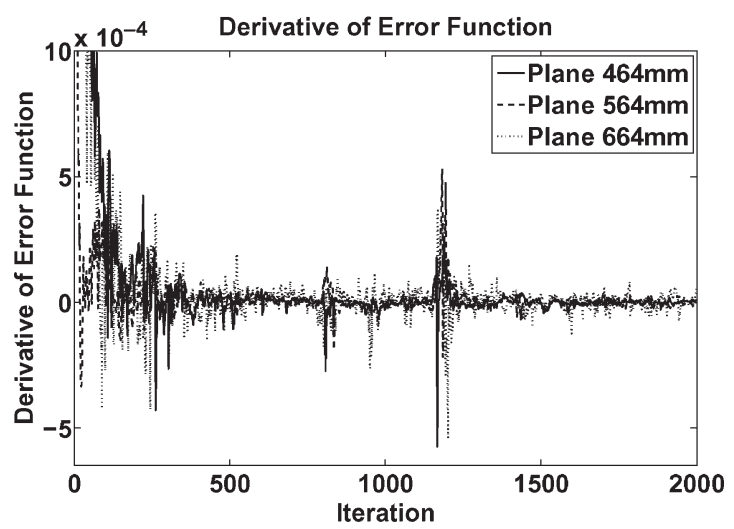

(d)

Fig. 10. Error function derivative calculated by (7) during the IPRA corresponding to the reconstruction efficiencies shown in Fig. 9. (a), (b), and (c) correspond to the different cases of HFP, while (d) is for the RSDI method. 


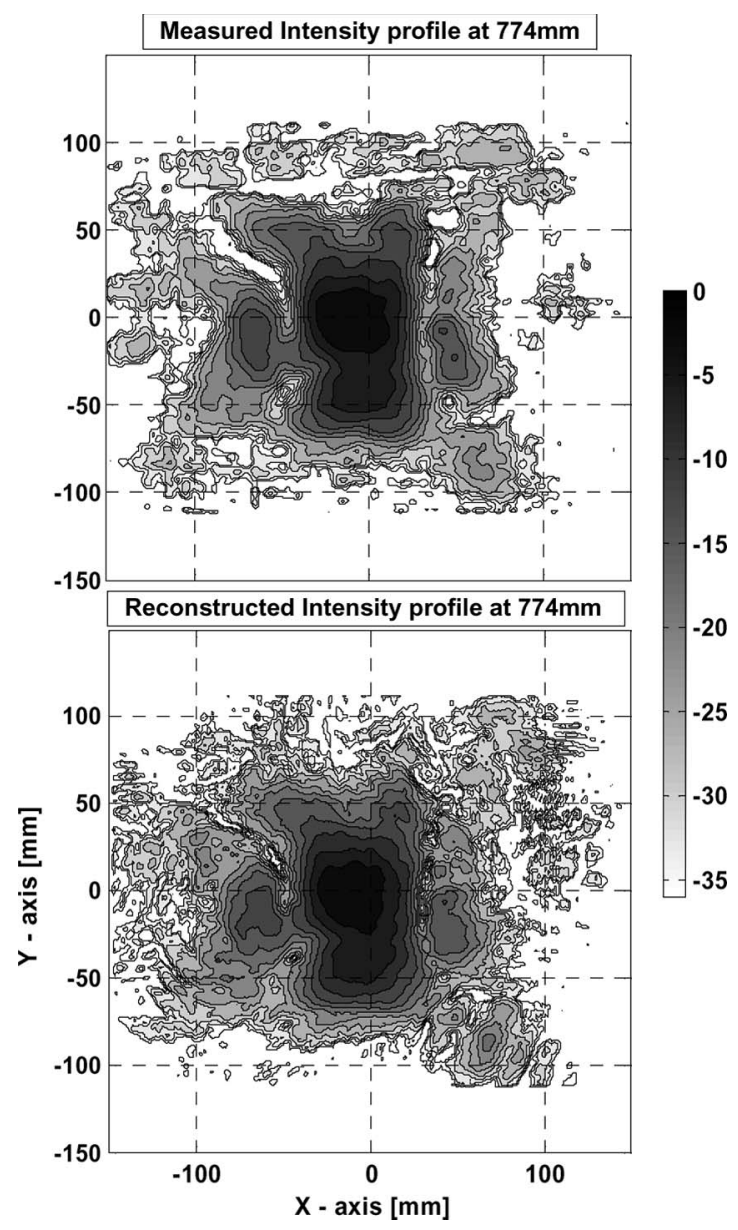

Fig. 11. Reconstruction check. (Top) Measured intensity pattern at $774 \mathrm{~mm}$ from the window. (Bottom) Intensity pattern by propagating the reconstructed field to $774 \mathrm{~mm}$. The contours are at every $-3 \mathrm{~dB}$ from the peak value. An excellent agreement can be seen between the measured and propagated fields using the reconstructed field.

measured intensity and theoretical intensity and phase profiles. This is shown in Fig. 11, where we showed the propagated intensity and measured intensity profiles at $764 \mathrm{~mm}$. The two intensity patterns match with each other down to a level of $\sim-27 \mathrm{~dB}$.

\section{B. Effect of Scan Separation, Number of Sampling Points, and Plane Size}

Based on the reconstructed results of the previous section where we concluded that the RSDI and HFP, with a fixed distance of propagation given by (4) and (5), produce accurate results, we therefore compare, using HFP in the IPRA, the reconstructed phase and intensity for two different cases of plane separation and number of sampling points. Fig. 12 shows the reconstructed intensity/phase and measured intensity using three planes at $464 / 564 / 664 \mathrm{~mm}$ (plane size of $300 \mathrm{~mm} \times$ $225 \mathrm{~mm}$ ) and 464/764/1122 $\mathrm{mm}$ (plane size of $300 \times 300 \mathrm{~mm}^{2}$ ) from the gyrotron window, respectively, in the IPRA. The reconstructed fields are compared at a common plane at $464 \mathrm{~mm}$. Intensities of the different cases are shown on a logarithmic scale, and the phase is shown in radians. It can be seen clearly that the reconstructed intensity calculated with the smaller

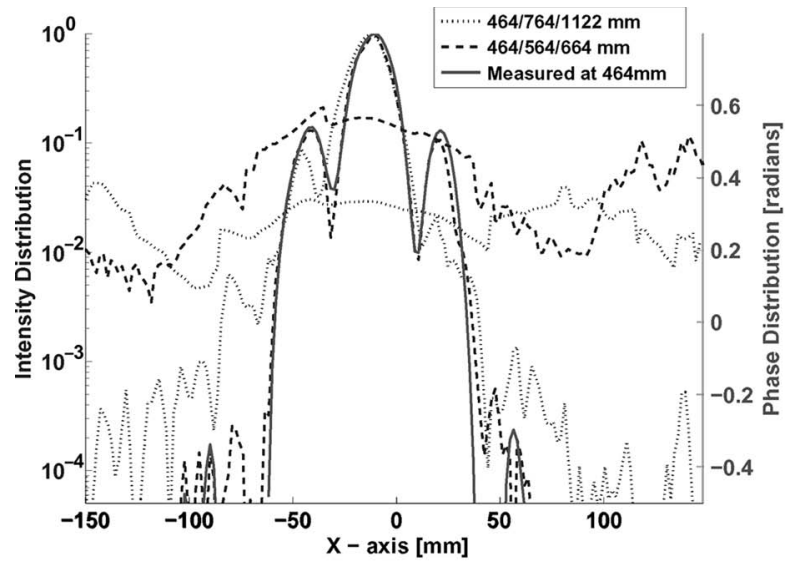

(a)

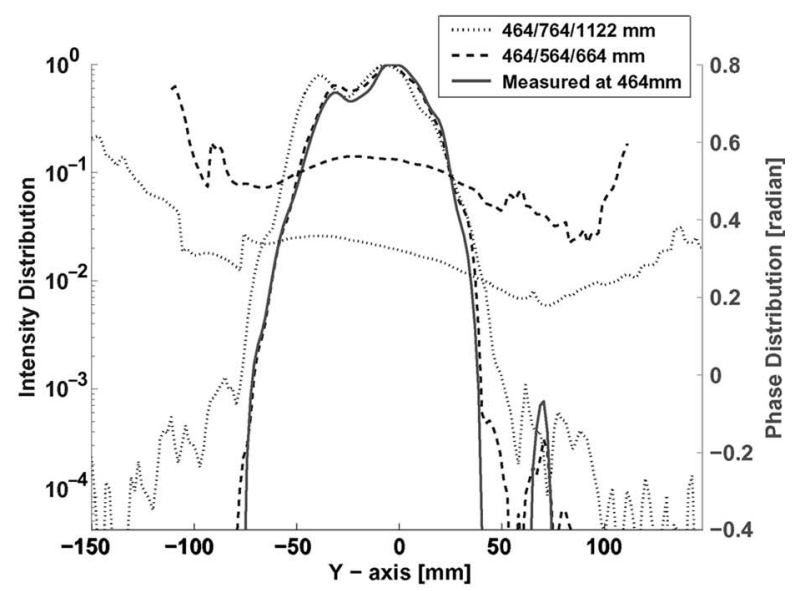

(b)

Fig. 12. Measured intensity and reconstructed intensity/phase distribution in the $x$ - and $y$-directions along the beam centroid $\langle x\rangle$ and $\langle y\rangle$. The reconstruction is done for two cases using planes at 464/564/664 and 464/764/1122 mm, respectively. (b) Phase plot for the first case is on a plane size of $300 \mathrm{~mm} \times$ $225 \mathrm{~mm}$ ( $225 \mathrm{~mm}$ on the $y$-axis), where the axis is shown as $300 \mathrm{~mm}$.

scan separation agrees with the measured intensity better than the larger scan separation. Moreover, the phase curvatures are different for the two scenarios. In the case of $464 / 764 / 1122 \mathrm{~mm}$, the field at the first plane is down interpolated to $M=170$ from $320 \times 240$, on a square plane of size $300 \mathrm{~mm} \times 300 \mathrm{~mm}$, to $M=143$ at plane 2 and to $M=78$ at the third plane during each iteration in order to follow the condition of propagation, i.e., (4). The field was propagated as $1 \rightarrow 2 \rightarrow 3$ in the forward direction and $3 \rightarrow 1$ in the backward direction. Since the measurement plane has the same size at every location and the field expands along the direction of propagation, we do not satisfy the constraint of field amplitude, which is zero at the boundary on the farther planes. Therefore, the field amplitude at the boundary is close to zero at the first plane while it is not at the second and third planes; hence, energy conservation is not followed during the iteration process. In addition, a large change in the number of sampling points at different planes leads to numerical errors, which is shown in Fig. 13 where we observe a random behavior in the $\mathrm{CCF}$, and the difference between the minimum and the maximum value is 0.15 . Fig. 13 is shown for the second case, i.e., larger scan separation where the planes are located at 464/764/1122 mm. A larger scan separation restricts one to use less sampling points for a fixed 


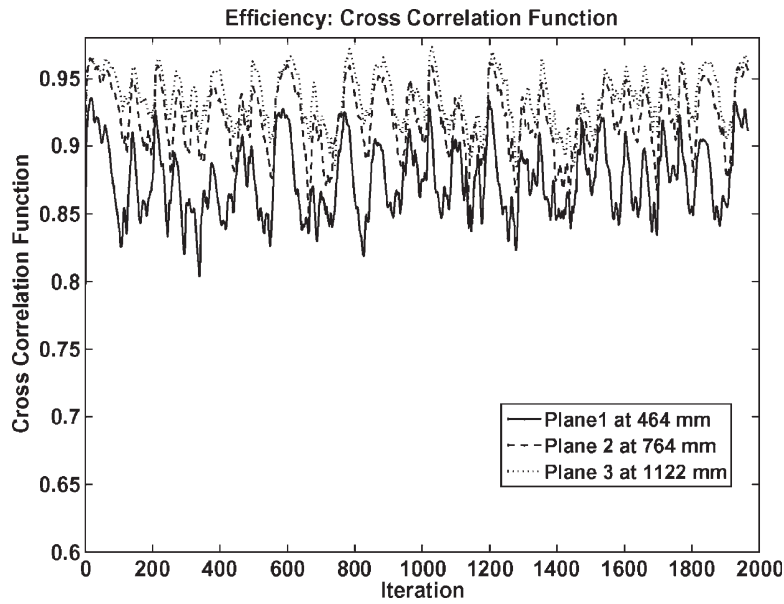

Fig. 13. CCF calculated during the IPRA for three planes at $464 / 764 / 1122 \mathrm{~mm}$.

plane size. This can be easily analyzed by (4). This restriction (having lesser sampling points) leads to a less accurate result and to the erroneous convergence of the algorithm. This is also explained in [8] in detail, where (4) is plotted for different numbers of sampling points. Furthermore, since the error function derivative should reach the saturation level monotonically and should be terminated at its global minimum, a predetermined value of error termination can end the iteration process at some wrong value. This is shown in Fig. 13 if one terminates the algorithm at a lower value which has been fixed earlier.

\section{CONCLUSION}

A detailed approach of IR measurements of the gyrotron output microwave beam and their data processing was studied and presented. In addition, the reconstruction process was analyzed with two propagation schemes in terms of sampling problems and other parameters. It has been observed that Robax can sustain higher temperatures and can therefore give intensity profiles with a larger dynamic range. Moreover, the difference in the effective beam waist is less in the case of Robax than PVC if measured from the front or back side. On the other hand, Robax is transparent to IR radiation. Interference effects, due to different reflections between the window (and its support) and target material, and Fabry-Parot effects inside the target material should be avoided. Accurate phase reconstruction requires an almost zero $(\sim 60 \mathrm{~dB})$ field amplitude at the boundary; therefore, it is better to go closer to the beam-waist position (also reveals more details if one goes closer to the beam waist as in the obvious in case of the 170-GHz/2-MW gyrotron output beam) as it will require a smaller target plane size. In addition, the relative phase evolution of modes in a higher order mode mixed field is more significant in the regions closer to the beamwaist location.

A tradeoff between the two methods of field propagation, the RSDI, and the HFP is needed. The RSDI method for field propagation is more convenient as it can be used for any number of sampling points and grid step size, and it is not restricted by the condition of propagation. Moreover, it does not require field interpolation to a different grid at every iteration. However, it is more computationally demanding. HFP requires field pattern measurements at specific locations, or an interpolation of field to a different grid size is necessary to satisfy the condition of propagation in order to avoid the aliasing effects.

Further modifications can be made to the IPRA by introducing a correction term in the iteration process by using the correlation between phase gradients and intensity of the field or by using an unwrapping technique to unwrap and smooth the phase at every iteration. These topics could be the subjects of future research.

\section{ACKNOWLEDGMENT}

The authors would like to thank Jean-Claude Magnin for the design and construction of the RF Box which was used for the measurements.

\section{REFERENCES}

[1] J. P. Hogge, S. Alberti, A. Arnold, D. Bariou, P. Benin, T. Bonicelli, A. Bruschi, R. Chavan, S. Cirant, O. Dumbrajs, D. Fasel, F. Gandini, E. Giguet, T. Goodman, R. Heidinger, M. Henderson, S. Illy, J. Jin, C. Lievin, R. Magne, P. Marmillod, P. L. Mondino, A. Perez, B. Piosczyk, L. Porte, T. Rzesnicki, M. Santinelli, M. Thumm, M. Q. Tran, and I. Yovchev, "Development of a 2-MW, CW coaxial gyrotron at $170 \mathrm{GHz}$ and test facility for ITER," J. Phys.: Conf. Series, vol. 25, pp. 33-44, 2005.

[2] J.-P. Hogge, S. Alberti, F. Albajar, P. Benin, S. Bethuys, T. Bonicelli, S. Cirant, E. Droz, O. Dumbrajs, D. Fasel, G. Gantenbein, T. P. Goodman, S. Illy, S. Jawla, J. Jin, S. Kern, C. Lievin, B. Piosczyk, I. Pagonakis, L. Porte, T. Rzesnicki, U. Siravo, M. Thumm, and M. Q. Tran, "First experimental results from the EU 2 MW coaxial cavity ITER gyrotron prototype," Fusion Sci. Technol., Feb. 2009. to be published.

[3] S. Alberti, F. Albajar, K. A. Avramides, P. Benin, W. Bin, T. Bonicelli, A. Bruschi, S. Cirant, E. Droz, O. Dumbrajs, D. Fasel, F. Gandini, T. Goodman, J.-P. Hogge, S. Illy, S. Jawla, J. Jin, S. Kern, C. Lievin, B. Marlétaz, P. Marmillod, I. Pagonakis, A. Perez, B. Piosczyk, L. Porte, T. Rzesnicki, U. Siravo, M. Thumm, and M. Q. Tran, "Status of development of the $2 \mathrm{MW}, 170 \mathrm{GHz}$ coaxial-cavity gyrotron for ITER," in Proc. 33rd Int. Conf. Infrared, Millimeter, Terahertz Waves, IRMMW-THz Pasadena, CA, Sep. 15-19, 2008.

[4] A. V. Chirkov, G. G. Denisov, and N. L. Aleksandrov, "3D wavebeam field reconstruction from intensity measurements in a few cross sections," Opt. Commun., vol. 115, no. 5, pp. 449-452, Apr. 1995.

[5] H. Idei, T. Shimozuma, M. A. Shapiro, T. Notake, S. Kubo, and R. J. Temkin, "Experimental verification of phase retrieval of quasi-optical millimeter-wave beams," IEEE Trans. Microw. Theory Tech., vol. 54, no. 11, pp. 3899-3905, Nov. 2006.

[6] M. A. Shapiro, T. S. Chu, D. R. Denison, M. Sato, T. Shimozuma, and R. J. Temkin, "Design of correcting mirrors for a gyrotron used at large helical device," Fusion Eng. Des., vol. 53, no. 1, pp. 537-544, Jan. 2001.

[7] J. P. Anderson, M. A. Shapiro, R. J. Temkin, and D. R. Denison, "Phase retrieval of gyrotron beams based on irradiance moments," IEEE Trans. Microw. Theory Tech., vol. 50, no. 6, pp. 1526-1535, Jun. 2002.

[8] S. Jawla, J.-P. Hogge, and S. Alberti, "Theoretical investigation of iterative phase retrieval algorithm for quasi-optical millimeter wave RF beams," IEEE Trans. Plasma Sci. accepted for publication.

[9] S. Jawla, J.-P. Hogge, S. Alberti, T. Goodman, B. Piosczyk, T. Rzesnicki, and C. Liévin, "Beam profile measurements of the output microwave beam of $2 \mathrm{MW} / 170 \mathrm{GHz}$ coaxial-cavity gyrotron for ITER," in Proc. 35th IEEE ICOPS, Karlsruhe, Germany, 2008, p. 249.

[10] T. Rzesnicki, J. Jin, B. Piosczyk, M. Thumm, G. Michel, and D. Wagner, "170 GHz, $2 \mathrm{MW}$ coaxial cavity gyrotron-Design and experimental verification of the RF output system," in Proc. 6th IEEE IVEC, Noordwijk, The Netherlands, Apr. 2005, pp. 303-306.

[11] F. I. Shimabukuro, S. Lazar, M. R. Chernick, and H. B. Dyson, "A quasioptical method for measuring the complex permittivity of materials," IEEE Trans. Microw. Theory Tech., vol. MTT-32, no. 7, pp. 659-665, Jul. 1984.

[12] T. Aach and V. H. Metzler, "Defective pixel interpolation in digital radiography: How object oriented transform coding helps," Proc. SPIE, vol. 4322, p. 824, 2001.

[13] L. A. Vese and S. J. Osher, "Image denoising and decomposition with total variation minimization and oscillatory functions," J. Math. Imaging Vis., vol. 20, no. 1/2, pp. 7-18, Jan. 2004. 
[14] L. I. Rudin, S. Osher, and E. Fatemi, "Nonlinear total variation based noise removal algorithms," Physica, D, vol. 60, no. 1-4, pp. 259-268, Nov. 1992.

[15] A. Buades, B. Coll, and J. M. Morel, "A review of image denoising algorithms, with a new one," Multiscale Model. Simul., vol. 4, no. 2, pp. 490-530, 2005.

[16] A. K. Jain, Fundamentals of Digital Image Processing. Englewood Cliffs, NJ: Prentice-Hall, 1990.

[17] J. W. Goodman, Introduction to Fourier Optics. New York: McGrawHill, 1968

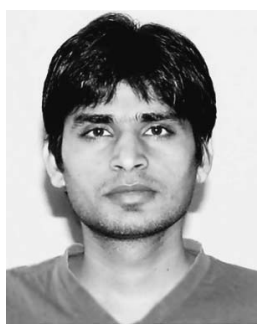

Sudheer Jawla (S'08) was born in Uttar Pradesh, India, in 1982. He received the B.Sc. degree from Chaudhary Charan Singh University, Meerut, India, in 2002 and the M.Sc. degree in physics from the Indian Institute of Technology, New Delhi, India, in 2004. Since 2005, he has been working toward the Ph.D. degree in high-power gyrotrons in the Gyrotron Research Group, Centre de Recherché en Physique des Plasmas, Association EuratomConfédération Suisse, École Polytechnique Fédérale de Lausanne, Lausanne, Switzerland.

His research interests include quasi-optical millimeter-wave components for high power gyrotrons and, laser and microwave driven particle acceleration.
Jean-Philippe Hogge, photograph and biography not available at the time of publication.

Stefano Alberti, photograph and biography not available at the time of publication.

Timothy Goodman, photograph and biography not available at the time of publication.

Bernhard Piosczyk, photograph and biography not available at the time of publication.

Tomasz Rzesnicki, photograph and biography not available at the time of publication. 